\title{
激光多普勒测速圆环壁面窗口折射效应的影响“
}

\author{
李春明 ${ }^{1}$ 李 晋 ${ }^{1}$ 李慎龙 $^{1,2}$ 魏 巍 ${ }^{3}$
}

(1. 中国北方车辆研究所 北京 100072;

2. 北京理工大学北京电动车辆协同创新中心 北京 100081 ;

3. 北京理工大学机械与车辆学院 北京 100081)

\begin{abstract}
摘要: 采用转盘标定系统和理论分析, 对激光多普勒测速仪(Laser Doppler anemometer, LDA)圆环壁面窗口折射效应进行研究, 建立描述两束激光轨迹的折射效应理论计算模型, 应用此模型推导了测量体位置和速度矢量偏差修正公式, 分析不同光束半 夹角 $\alpha_{1}$ 、内流场介质折射率 $n_{3}$ 、圆环壁面厚度 $d$ 、探头位置对折射效应的影响。应用转盘标定系统对计算模型进行验证, 并 且进行了条纹畸变折射效应研究。使用相对平移系数 $k_{x} 、 k_{y}$ 和速度误差 $\Delta u$ 来评估折射效应对于测量体位置和测速误差的影 响。结果表明: 控制 $\alpha_{1}$ 在 $10^{\circ}$ 以内可以忽略其折射效应影响; 壁厚 $d=30$ 时, 折射效应产生的 $k_{x} 、 k_{y}$ 和 $\Delta u$ 近似为恒定值; 壁 面两侧折射率尽可能的接近, 可减小 $n_{3}$ 的折射效应; $\Delta u$ 试验数据略小于计算数据, 结果相对比较吻合。
\end{abstract}

关键词: 激光多普勒测速; 圆环壁面; 测量体偏移; 条纹畸变; 标定转盘

中图分类号: TH137; TH249

\section{Refraction Effect from Beam Refraction by Annular Wall Window in LDA Applications}

\section{Chunming $^{1} \quad$ LI Jin $^{1} \quad$ LI Shenlong, ${ }^{1,2}$ WEI Wei ${ }^{3}$}

(1. China North Vehicle Research Institute, Beijing 100072;

2. Collaborative Innovation Center of Electric Vehicles in Beijing, Beijing 100072;

3. School of Mechanical Engineering, BeijingInstitute of Technology, Beijing 100081)

\begin{abstract}
The influence of laser beam refraction resulting from the annular wall window on refraction effect is investigated both in a theoretical analysis and laser Doppler anemometry(LDA) using a calibration disc. A mathematical procedure, without any simplifications, describing the propagation of two laser beams through an annular window was established to study the effect of refraction on measuring volume(MV) shift in terms of laser beam half angle, annular window thickness, refractive index of the fluid, and LDA probe location. A test system used for LDA calibration was assembled to study this effect of refraction to fringe distortion. The coefficients of relative motion $k_{x}$ and $k_{y}$, and the velocity bias $\Delta \mathrm{u}$ relative to the real fluid velocity are used to estimate the refraction influence on MV position and velocity bias. Results show that the effect can be ignored within $10^{\circ}$ of the half angle. $k_{x}, k_{y}$, and $\Delta \mathrm{u}$ are nearly constant if the thickness of the annular wall window is more than $30 \mathrm{~mm}$. The refractive index of the fluid should be matched as near possible with that of the annular wall to minimalize the refractive influence. The velocity bias arising from the change in fringe spacing is smaller than that estimated by the mathematical procedure.
\end{abstract}

Key words: laser doppler anemometer; annular wall window; measurement volume shift; fringe distortion; calibration disk

\section{0 前言}

对液力变矩器(图 1)内流场的激光多普勒测速仪 (Laser Doppler anemometer, LDA)测试，激光光束需 要穿越叶轮圆环壁面, 改变了原有测量体位置, 进而

\footnotetext{
* 坦克传动国防科技重点实验室基金资助项目(9140C340204150C34153)。
} 20161202 收到初稿, 20170628 收到修改稿
对 LDA 测量产生诸多不利影响: 测速位置发生偏移; 测速方位和大小发生改变; 多普勒信号强度和数据有 效率下降。多维探头受其影响更严重，可能导致多维 光束聚焦困难，无法实现多维实时协同测量。

平板窗口是 LDA 实际应用所遇到最多的情况, 因此国内外对于 LDA 折射效应的研究就主要集中 于平板折射效应, 徐兴祺等 ${ }^{[1-7]}$ 均对 LDA 平板折射 效应进行了研究, 包括激光斜入射情况, 推导了测 量体折射后的位置修正模型，尤其是赵军等 ${ }^{[4]}$ 还研 
究了二维探头蓝光和绿光测量体经过平板折射效应 不重合的慧差现象。

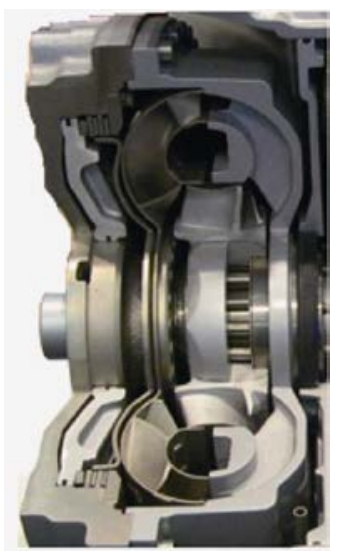

图 1 液力变矩器

而对于曲面 LDA 折射效应的研究集中于管道壁 面(即圆柱壁面)折射效应，除了进行折射率匹配 ${ }^{[8-10] 、}$ 管道壁面加窗 ${ }^{[5]}$ 等物理修正方法减小折射效应的影响 外, 许多学者对管道壁面折射效应进行了理论计算。 徐兴祺 ${ }^{[1]}$ 对激光光束垂直入射 0 厚度圆柱壁面情况 下, 折射效应对测量体位置的影响进行了理论推导。 ZHANG 等 ${ }^{[5,11]}$ 研究了激光光束垂直和斜入射圆柱壁 面对测量体位置、条纹问距、所测速度方向和速度误 差的影响, 并同平板窗口分析一样, 着重分析了斜入 射角度和平板厚度对二维探头蓝绿测量体间距的影 响。RISTIC 等 ${ }^{[12]}$ 研究了不同壁厚、半径和光束半夹 角对 LDA 探头光轴非垂直入射圆柱壁面管道中测量 体位置及速度方向的影响。DOUKELIS 等 ${ }^{[13]}$ 通过编制 相应的计算程序, 研究了激光光束垂直和斜入射圆柱 壁面下, 不同圆柱壁面曲率、壁厚和测试深度对测量 体位置的影响，并对其程序进行了试验验证。

而对于呈圆环形的旋转机械壁面的折射效应未 见有学者做相关研究。本文以液力变矩器泵轮壁面 为研究对象, 采用 LDA 测试系统二维探头, 进行不 同光束半夹角、介质折射率、和壁面厚度对测量体 位置和速度矢量影响的研究, 用以指导试验参数的 选取、方案的确定和测速结果的修正。

\section{1 折射效应计算模型}

应用 LDA 测试系统二维探头进行圆环壁面内 流场测试时, 如图 2 所示, $x$ 轴为圆环壁面旋转轴, $O x y$ 平面为水平平面即二维探头的绿光平面, $O y z$ 平面为坚直平面即平行于蓝光平面。激光光束经过 三种不同介质(折射率分别为 $n_{1} 、 n_{2}$ 和 $n_{3}$ ), 圆环壁 面在 $O x y$ 平面上的圆心为 $P_{0}$, 半径为 $r$, 壁厚为 $d$ 。 若无折射效应绿光和蓝光初始交于 $C$, 光束半夹角
均为 $\alpha_{0}$ 。经过折射后绿光交于 $M$, 光束半夹角为 $\alpha_{1}$, 蓝光交于 $S$, 光束半夹角为 $\alpha_{2}$ 。由于圆环面对称, 则仅对 $x$ 轴负方向进行分析。具体折射效应计算模 型和计算流程如下所述。

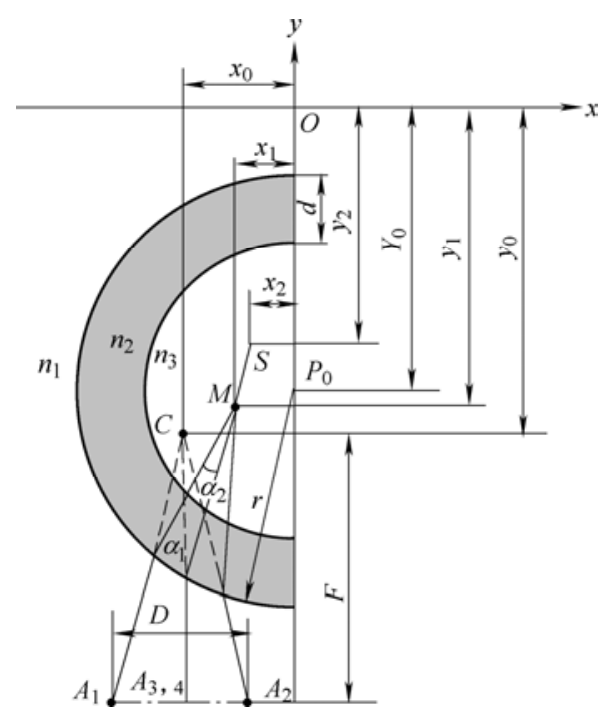

图 2 圆环壁面计算模型

\section{1 求解入射光线及初始光束半夹角}

进行空间 LDA 折射效应研究时, 首先应确定入 射光线的空间方程，为方便下一步求解入射光线在 圆环面上的入射点, 射线方程采用空间参数方程

$$
\left\{\begin{array}{l}
x=X_{0}+t l \\
y=Y_{0}+t m \\
z=Z_{0}+t n
\end{array} \quad t \geqslant 0\right.
$$

式中, $t$ 为参数, $A\left(X_{0}, Y_{0}, Z_{0}\right)$ 为射线起始点, $r=(l, m, n)$ 为射线单位方向矢量。设绿光光束对起 始点为 $A_{1}$ 和 $A_{2}$, 蓝光光束对起始点为 $A_{3}$ 和 $A_{4}$, 如 图 2 所示, 通过给定初始交点 $C$ 坐标 $\left(x_{0}, y_{0}, z_{0}\right)$ 、探 头焦距 $F$, 以及光束间距 $D$, 结合几何关系, 可得 四个光束起始点坐标为 $A_{1}\left(x_{0}-D / 2, y_{0}-F, z_{0}\right)$ 、 $A_{2}\left(x_{0}+D / 2, y_{0}-F, z_{0}\right) 、 A_{3}\left(x_{0}, y_{0}-F, z_{0}-D / 2\right)$ 和 $A_{4}\left(x_{0}, y_{0}-F, z_{0}+\right.$ $D / 2)$, 单位方向矢量 $\boldsymbol{r}_{i}$ 为

$$
r_{i}=\frac{A_{i} C}{\left|A_{i} C\right|}
$$

式中, $i=1,2,3,4$ 。

同时可以获得绿光和蓝光的初始光束半夹角, 均为 $\alpha_{1}$

$$
\alpha_{1}=\arctan \frac{D}{2 F}
$$

\section{2 求解入射点}

$O x y$ 平面上, 圆心坐标 $P_{0}$ 为 $\left(0, Y_{0}\right)$, 半径为 $r$ 的圆绕 $x$ 轴旋转一周, 所得圆环面方程为

$$
\left(x^{2}+y^{2}+z^{2}+Y_{0}^{2}-r^{2}\right)^{2}-4 Y_{0}^{2}\left(y^{2}+z^{2}\right)=0
$$


圆环面上任意一点 $P(x, y, z)$ 的法向矢量 $\boldsymbol{n}=\left(F_{x}, F_{y}, F_{z}\right)$, 这里

$$
\left\{\begin{array}{l}
F_{x}=4 x\left(x^{2}+y^{2}+z^{2}+Y_{0}^{2}-r^{2}\right) \\
F_{y}=4 y\left(x^{2}+y^{2}+z^{2}-Y_{0}^{2}-r^{2}\right) \\
F_{z}=4 z\left(x^{2}+y^{2}+z^{2}-Y_{0}^{2}-r^{2}\right)
\end{array}\right.
$$

将入射光线方程式(1)代入式(4), 可以获得关于 参数 $t$ 的一元四次方程, 对其进行求解, 取正实数 最小根重新代入方程式(1), 即可获得入射光线在圆 环面上的入射点坐标, 将其代入式(5)则可获得入射 点在圆环面上的法向矢量, 将其单位化为 $\boldsymbol{r}_{0}$, 用于 下一步求解折射光线空间方程。

\section{3 求解折射光线}

光线穿越折射率为 $n_{1}$ 和 $n_{2}$ 两种不同介质时会 发生折射现象, 结合折射定律可得折射光线单位方 向矢量

$$
\boldsymbol{r}_{1}^{\prime}=\frac{n_{1}}{n_{2}} \boldsymbol{r}_{1}-\left(\sqrt{1-\left(\frac{n_{1}}{n_{2}}\right)^{2}+\left(\frac{n_{1}}{n_{2}} \boldsymbol{r}_{1} \cdot \boldsymbol{r}_{0}\right)^{2}}+\frac{n_{1}}{n_{2}} \boldsymbol{r}_{1} \cdot \boldsymbol{r}_{0}\right) \boldsymbol{r}_{0}
$$

式中, $\boldsymbol{r}_{1}$ 为入射光线单位方向矢量, $\boldsymbol{r}_{0}$ 为两介质交 界面入射点处单位法向矢量。将 $\boldsymbol{r}_{1}^{\prime}$ 和由 2.2 求出的 入射点坐标一同代入式(1), 可获得空间折射光线。

\section{4 求解折射后交点、光束半夹角和速度偏角}

因光线折射需经过两层圆环面, 将第 2.3 节中 求解的折射光线作为第二层圆环面 $(O x y$ 平面圆半 径为 $\left.r^{\prime}=r-d\right)$ 的入射光线, 重复第 2.2 节和第 2.3 节 的计算, 获得最终折射光线空间方程, 联立一对折 射光线空间方程即可获得折射后的交点坐标。

并由这对折射光线单位方向矢量 $\boldsymbol{r}_{1}^{\prime}$ 和 $\boldsymbol{r}_{2}^{\prime}$, 可获 得折射后光束半夹角 $\alpha_{2}$ 和速度方向矢量 $\boldsymbol{v}_{2}^{\prime}$

$$
\left\{\begin{array}{l}
\alpha_{2}=\frac{\arccos \left|\boldsymbol{r}_{1}^{\prime} \cdot \boldsymbol{r}_{2}^{\prime}\right|}{2} \\
\boldsymbol{v}_{2}^{\prime}=\left(\boldsymbol{r}_{1}^{\prime} \times \boldsymbol{r}_{2}^{\prime}\right) \times\left(\boldsymbol{r}_{1}^{\prime}+\boldsymbol{r}_{2}^{\prime}\right)
\end{array}\right.
$$

结合未经折射的初始测速方向矢量 $v_{1}^{\prime}$ 可获得速 度偏角

$$
\beta=\frac{\arccos \frac{\left|\boldsymbol{v}_{1}^{\prime} \cdot \boldsymbol{v}_{2}^{\prime}\right|}{\left|\boldsymbol{v}_{1}^{\prime}\right| \cdot\left|\boldsymbol{v}_{2}^{\prime}\right|}}{2}
$$

\section{2 试验验证}

\section{1 试验装置}

基于折射效应计算模型, 建立了转盘 LDV 标 定试验台, 如图 3 所示, 被试圆环壁面透明样件
固定于光学平台上, $\mathrm{LDA}$ 二维探头固定于光学平 台上的升降平台, 用于调整其与试验样件的相对 位置。标定转盘固定于平台上的电动三维坐标架, 用以捕获经圆环壁面折射后, 激光光线交点的位 置。转盘内 $5 \mu \mathrm{m}$ 的铇丝用于模拟测试区域中的失 踪粒子。

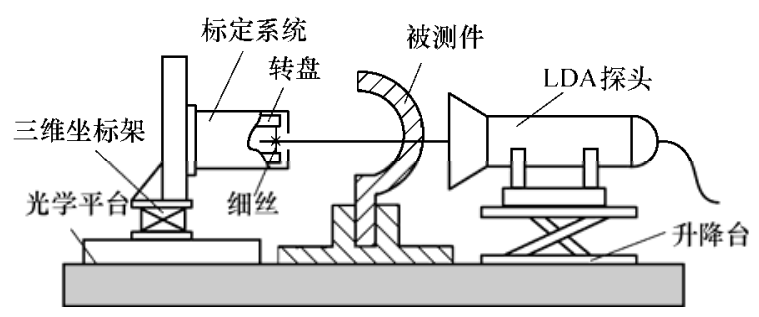

图 3 试验台组成示意图

升降平台的 LDA 测试探头沿 $y$ 方向改变测量体 的位置, 具体测试位置划分如图 4 所示。试验中的 系统参数见表 1 。

\begin{tabular}{|c|c|c|c|c|}
\hline $\begin{array}{l}\text { 有机玻璃 } \\
\text { 折射率 } n_{2}\end{array}$ & $\begin{array}{c}\text { 光速初始 } \\
\text { 半夹角 } \alpha_{0} /\left(^{\circ}\right)\end{array}$ & $\begin{array}{c}\text { 透镜焦距 } \\
l_{f} / \mathrm{mm}\end{array}$ & $\begin{array}{c}\text { 光速直径 } \\
D_{f 1} / \mu \mathrm{m}\end{array}$ & $\begin{array}{c}\text { 激光波长 } \\
\lambda_{1} / \mathrm{nm}\end{array}$ \\
\hline 1.517 & 2.768 & 2.49 & 5.327 & 514.5 \\
\hline $\begin{array}{c}\text { 透镜焦距 } \\
f / \mathrm{mm} \\
\end{array}$ & $\begin{array}{c}\text { 光速间距 } \\
D / \mathrm{mm}\end{array}$ & $\begin{array}{c}\text { 壁面厚度 } \\
d / \mathrm{mm} \\
\end{array}$ & $\begin{array}{c}\text { 标定转速 } \\
u_{\mathrm{w}} /(\mathrm{m} / \mathrm{s})\end{array}$ & \\
\hline 800 & 77.36 & 15 & 7.338 & \\
\hline
\end{tabular}

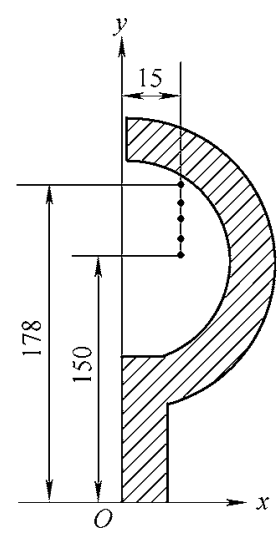

图 4 试验测试点示意图

表 1 试验系统参数表

\section{2 试验步骤}

通过判断由 LDA 系统测试软件获得的多普勒 波形, 可以确定测量体是否聚焦到细丝上, 如图 5 所示, 每隔 $0.2 \mathrm{~mm}$ 移动装盘标定系统位置, 使细丝 横扫整个测量体, 并同时记录下细丝位置坐标和相 应的多普勒信号。

实际测量体条纹间距 $d_{f 2}$ 可由标定转盘细丝的 标准圆周切向分速度 $u_{w}$ 和实际测得的多普勒频率 $f_{D}$ 获得

$$
d_{f 2}=\frac{u_{w}}{f_{D}}
$$




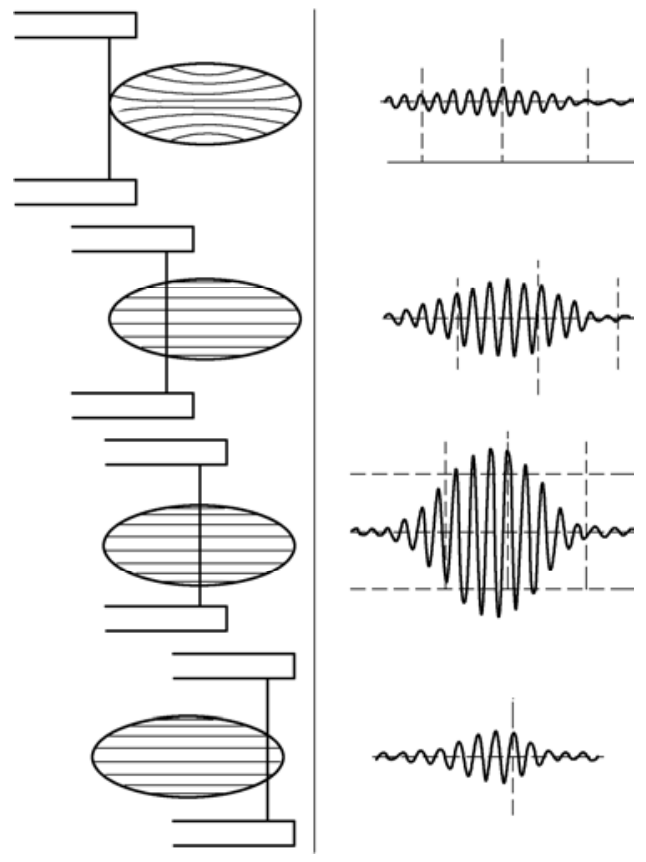

图 5 细丝位置与多普勒波形示意图

通过将实测测量体长度除以理论测量体长 度(表 1 ), 得到归一化的测量体长度后, 在每个 图 5 所示的测量位置重复上述测量和计算过程, 并应用理论测量体条纹间距 $d_{f 1}$, 获得折射后的速 度误差

$$
\Delta u=\left(\frac{d_{f 1}}{d_{f 2}}-1\right) \times 100 \%
$$

\section{3 试验结果}

无折射效应的测量体条纹间距如图 6 所示, 代 入到式(10), 获得速度误差 $\Delta u$ 曲线如图 7 所示, 可 知未经折射效应的速度误差平均值为 $0.49 \%$ 。

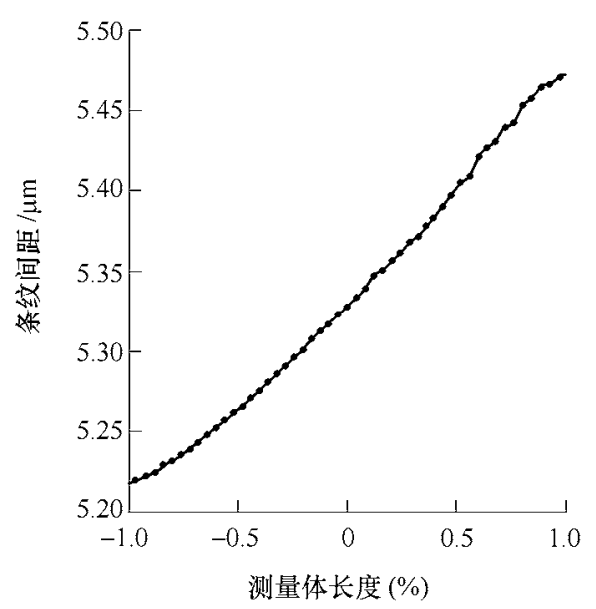

图 6 测量体条纹间距曲线

由于折射效应产生的不同位置的速度误差 $\Delta u$ 见图 8。随着探头位置从 $150 \mathrm{~mm}$ 到 $178 \mathrm{~mm}$, 由折 射效应计算程序获得的速度误差近似, 由计算和试 验获得的速度误差比较吻合, 误差在 5\%以内。

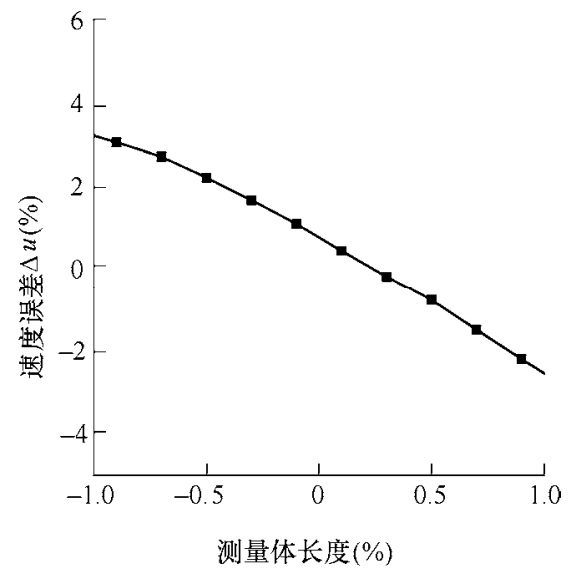

图 7 测量体速度误差曲线

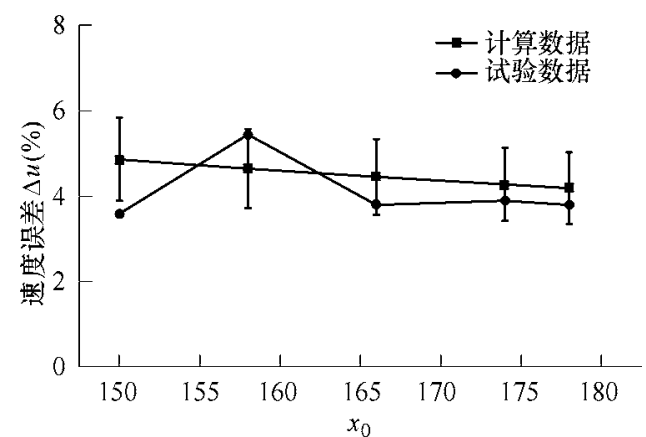

图 8 不同位置速度误差曲线

\section{3 折射效应分析}

应用上述折射效应计算模型，按照表 2 对圆环 壁面窗口折射效应进行计算，获得绿光和蓝光测量 体折射后位置如图 9 所示。

表 2 圆环壁面 LDA 折射效应模型参数表

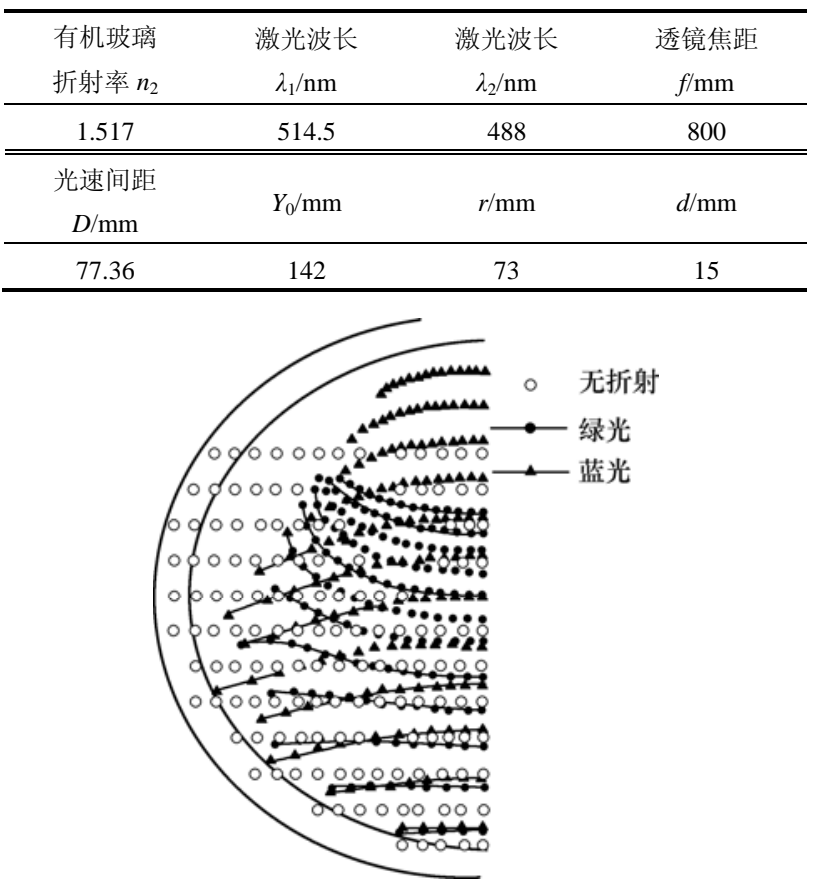

图 9 折射后测量体位置 
随着测试探头沿着 $x$ 轴移动, 折射后的测量体位 置会有 $x$ 和 $y$ 两个方向的相对移动, 此相对移动可以 用相对平移系数表征, 即 $k_{x x}$ 和 $k_{y x}$ (简写为 $k_{x}$ 和 $\left.k_{y}\right)^{[6]}$

$$
\left\{\begin{array}{l}
k_{x x}=\frac{\mathrm{d} x}{\mathrm{~d} x_{0}} \\
k_{y x}=\frac{\mathrm{d} y}{\mathrm{~d} x_{0}}
\end{array}\right.
$$

如果 $k_{x}$ 或是 $k_{y}$ 等于 1 , 那么代表折射后的测量 体位置相对移动量等同于测试探头的相对移动量, 即三维坐标架的坐标可以代表测量体在测试区域中 的位置。如果 $k_{x}$ 或 $k_{y}$ 小于 1 , 则代表测量体在测试 区域中相对移动的距离小于坐标架实际移动的距 离。如果 $k_{x}$ 或 $k_{y}$ 小于 0 , 则代表测量体在测试区域 中移动的方向与坐标架实际移动的方向相反。

通过速度偏移角随测试探头位置变化曲线可以 看出, 如图 10 所示, 速度偏移角仅受测试探头 $x$ 方 向移动的影响, 因此本文以下研究均以测试探头 $y$ 方向坐标恒定, 即 $y_{0}=120 \mathrm{~mm}$ 。

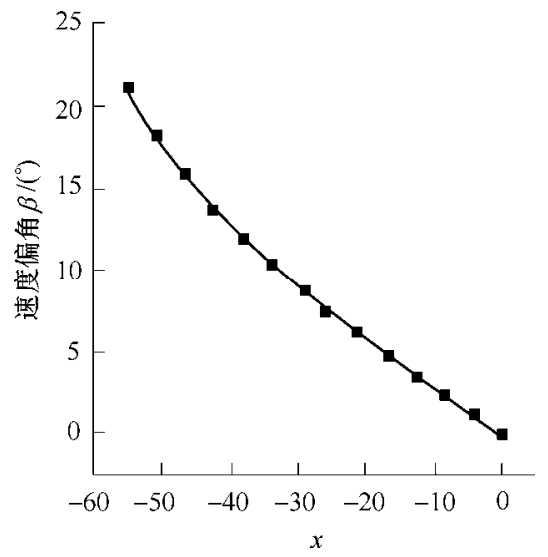

图 10 速度偏移角随测试探头位置变化曲线

应用折射效应计算模型，继续分析不同壁厚 $(d=10 \mathrm{~mm} 、 20 \mathrm{~mm} 、 30 \mathrm{~mm} 、 40 \mathrm{~mm}$ 和 $50 \mathrm{~mm})$ 、光 束半夹角 $\left(\alpha_{0}=2.17^{\circ} 、 3.4^{\circ} 、 5.43^{\circ} 、 7.73^{\circ}\right.$ 和 $\left.13.36^{\circ}\right)$ 和 测试区域介质折射率 $\left(n_{3}=1 、 1.3 、 1.5\right.$ 和 1.7$)$ 下的圆

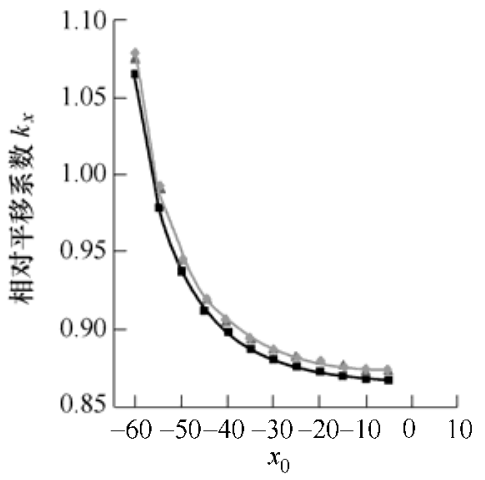

(a)

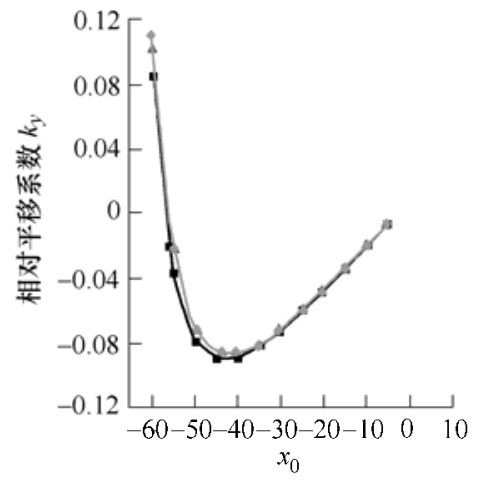

(b)
环壁面 LDA 折射效应, 对测量体位置和所测速度矢 量的影响。

\section{1 绿光折射效应影响}

光束半夹角对于相对平移系数和速度误差的影 响较小, 如图 11 所示, 并且当其在 $10^{\circ}$ 以内时的影 响可以忽略。随着 LDA 测试探头从壁面边缘移至壁 面中心, 相对平移系数 $k_{x}$ 从 1.07 逐渐降到 0.87 , 如 图 11a 所示。由于 $x$ 方向为测量体主要移动方向, 相对平移系数 $k_{y}$ 相对变化较小, 如图 $11 \mathrm{~b}$ 所示从 0.11 逐渐降至 -0.10 , 在越过 $x_{0}=-45$ 后逐渐上升至 0 (圆 环壁面中心位置)。对于速度偏差 $\Delta u$, 测试探头位 置的影响较大, 从-7\%增加到 15\%, 如图 11c, 即 探头位于壁面中心位置的速度误差最大。

随着圆环壁面厚度的增加, 相对平移系数 $k_{\mathrm{x}}$ 逐 渐减小, 速度误差 $\Delta u$ 逐渐增加, 如图 12 所示。当 $d<30 \mathrm{~mm}$ 时, 随着探头向圆环壁面中心的移动, $k_{\mathrm{x}}$ 逐渐减小。当 $d>30 \mathrm{~mm}$ 时, 随着探头向圆环壁面中 心的移动, $k_{x}$ 逐渐增加。当 $d=30 \mathrm{~mm}$ 时, $k_{x}$ 可以近 似等于 0.84 , 而不受探头位置的影响; 当 $d<30 \mathrm{~mm}$ 时, 随着探头向圆环壁面中心的移动, $k_{\mathrm{y}}$ 先逐渐减小 而后逐渐增加至 0 , 如图 12b 所示, 当 $d>30 \mathrm{~mm}$ 时, 随着探头向圆环壁面中心的移动, $k_{x}$ 逐渐增加, 并且 受 $d$ 的影响可以忽略; 类似对于 $k_{x}$ 的影响趋势, 但 折射效应对于速度误差 $\Delta u$ 的影响幅度更大, 如图 12c 所示。当 $d<30 \mathrm{~mm}$ 时, 随着探头向圆环壁面中心的 移动, $\Delta u$ 逐渐减小。当 $d>30 \mathrm{~mm}$ 时, 随着探头向圆 环壁面中心的移动, $\Delta u$ 逐渐增加。当 $d=30 \mathrm{~mm}$ 时, $\Delta u$ 可以近似等于 $19.7 \%$, 而不受探头位置的影响。

对于相对平移系数, 折射率的影响较为明显, 如图 13 所示。如果内流场折射率 $n_{3}>1$, 随着探头 向圆环壁面中心的移动, 相对平移系数 $k_{\mathrm{x}}$ 逐渐减小, $k_{\mathrm{y}}$ 先逐渐减小而后逐渐增加, 速度误差 $\Delta u$ 逐渐增 加。如果 $n_{3}=1$, 随着探头向圆环壁面中心的移动, 相对平移系数 $k_{x}$ 和 $k_{y}$ 逐渐增加, $\Delta u$ 逐渐减小。

图 11 不同光束半夹角下相对平移系数以及速度误差随测试探头位置变化影响曲线

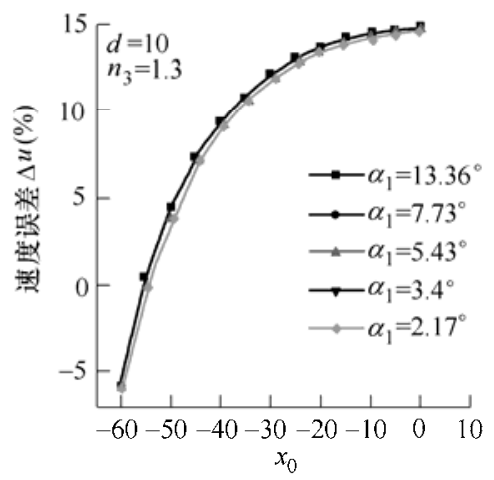

(c) 


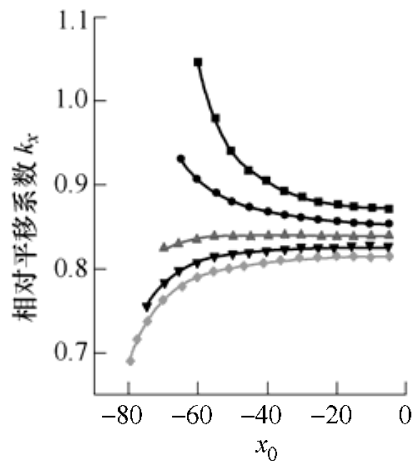

(a)

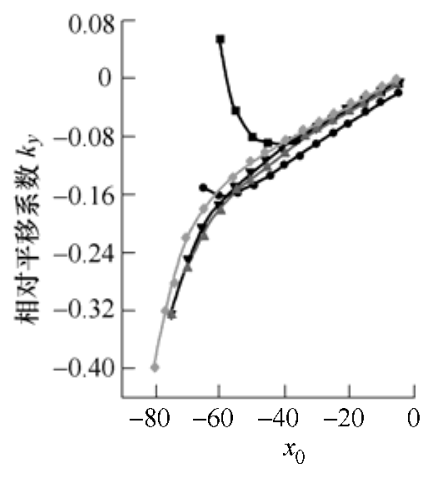

(b)

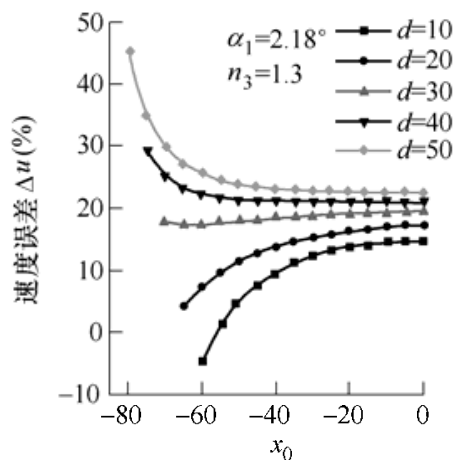

(c)

图 12 不同壁面厚度下相对平移系数以及速度误差随测试探头位置变化影响曲线

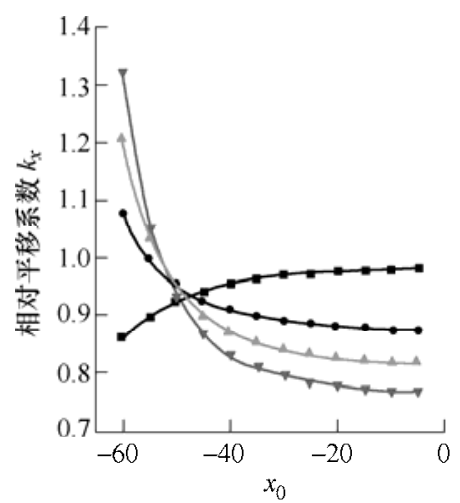

(a)

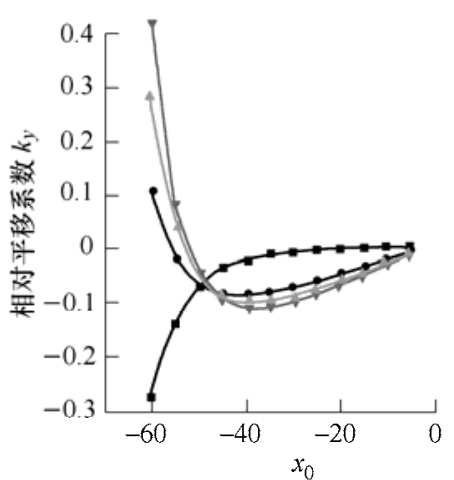

(b)

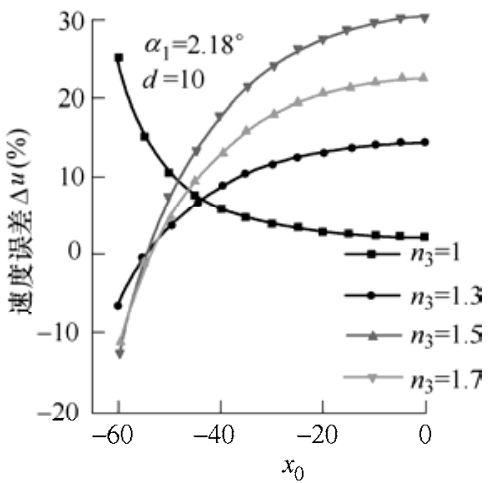

(c)

图 13 同介质折射率下相对平移系数以及速度误差随测试探头位置变化影响曲线

\section{2 蓝光折射效应影响}

光束半夹角对于相对平移系数和速度误差的影 响较小, 如图 14 所示, 并且当其在 $10^{\circ}$ 以内时的影 响可以忽略。随着 LDA 测试探头从壁面边缘移至壁 面中心, 相对平移系数 $k_{\mathrm{x}}$ 和 $k_{\mathrm{y}}$ 逐渐减小。对于速度 偏差 $\Delta u$, 测试探头位置的影响较大, 从 $1.6 \%$ 增加到 $5.4 \%$, 如图 14c 所示, 即探头位于壁面中心位置的 速度误差最大。

随着圆环壁面厚度的增加, 相对平移系数 $k_{x}$ 和 $k_{y}$ 逐渐减小, 速度误差 $\Delta u$ 逐渐增加, 如图 15 所示。 当 $d<30 \mathrm{~mm}$ 时, 随着探头向圆环壁面中心的移动, $k_{\mathrm{x}}$ 逐渐减小。当 $d>30 \mathrm{~mm}$ 时, 随着探头向圆环壁面 中心的移动, $k_{x}$ 逐渐增加。当 $d=30 \mathrm{~mm}$ 时, $k_{\mathrm{x}}$ 可以 近似等于 0.82 , 而不受探头位置的影响; 随着探头 向圆环壁面中心的移动, $k_{y}$ 逐渐减小至 0 , 如图 $15 \mathrm{~b}$ 所示; 随着探头向圆环壁面中心的移动, $\Delta u$ 逐渐增 加，如图 15c 所示。

如果内流场折射率 $n_{3}>1$, 随着探头向圆环壁面 中心的移动, 相对平移系数 $k_{x}$ 和 $k_{y}$ 逐渐减小, 速度 误差 $\Delta u$ 逐渐增加, 如图 16 所示。如果 $n_{3}=1$, 随着 探头向圆环壁面中心的移动, 相对平移系数 $k_{x}$ 和 $k_{y}$ 逐渐增加, $\Delta u$ 逐渐减小。

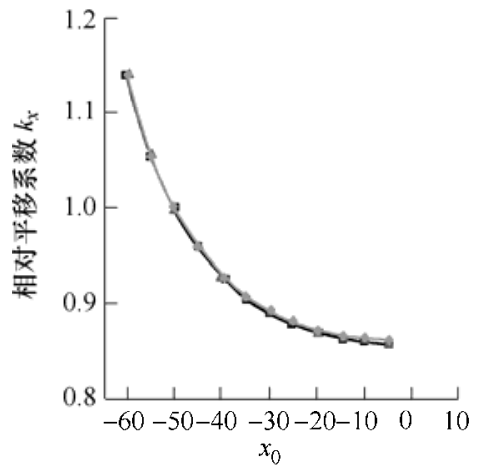

(a)

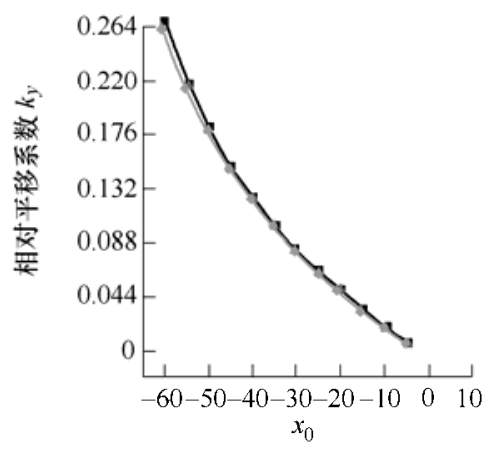

(b)

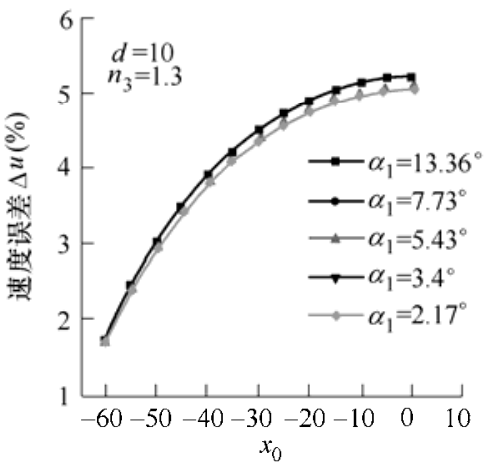

(c)

图 14 不同光束半夹角下相对平移系数以及速度误差随测试探头位置变化影响曲线 


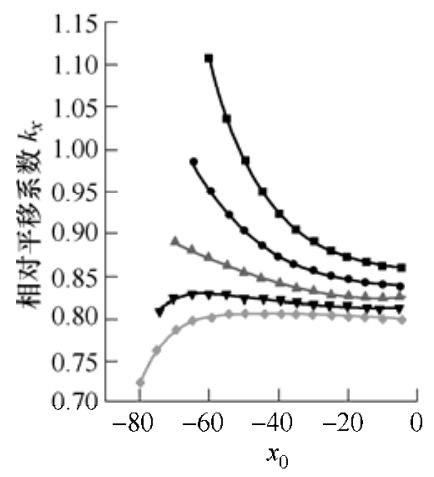

(a)

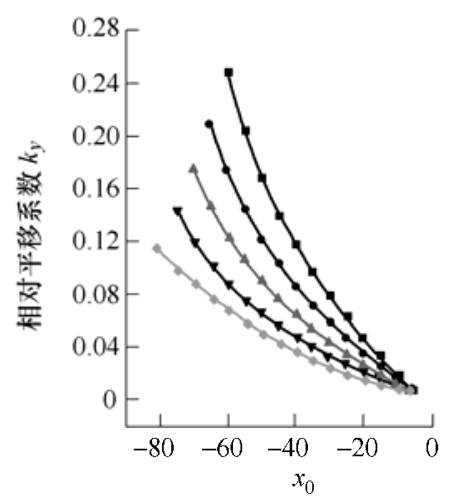

(b)

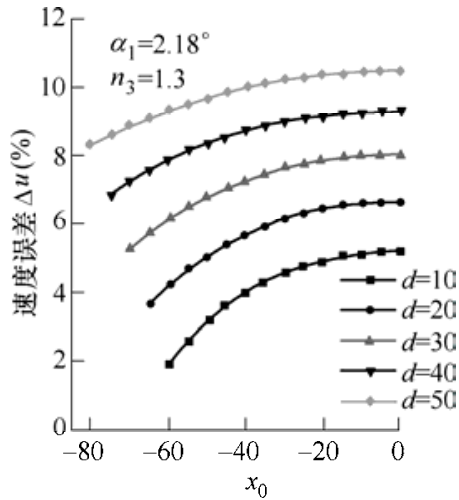

(c)

图 15 不同壁面厚度下相对平移系数以及速度误差随测试探头位置变化影响曲线

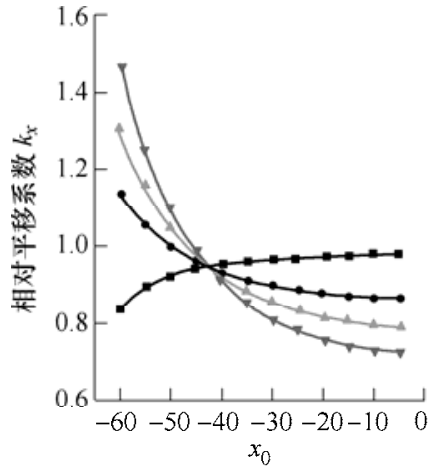

(a)

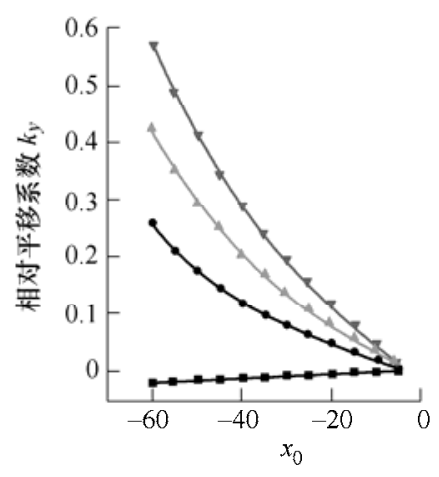

(b)

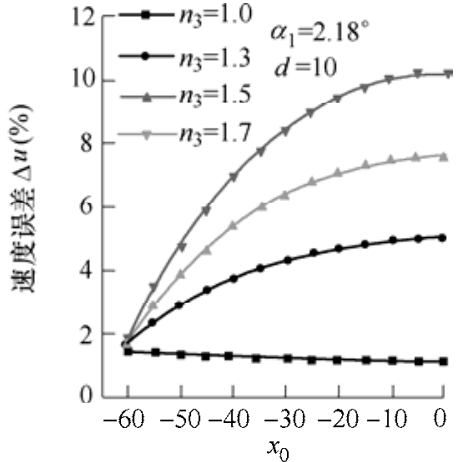

(c)

图 16 不同介质折射率下相对平移系数以及速度误差随测试探头位置变化影响曲线

\section{4 内流场测试实例}

通过修正后的激光多普勒测速(Laser Doppler anemometer, LDA)系统, 测试了泵轮转速 $800 \mathrm{r} / \mathrm{min}$, 速比 0.6 下的液力变矩器原轮中间平面内流场测试 数据, 测试区域如图 17 所示。

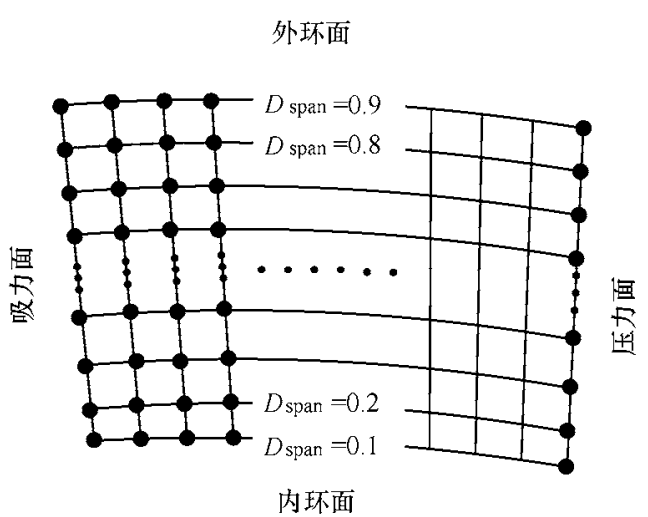

图 17 中间面示意图

同时将中间平面的 LDA 所测三维速度分布矢 量图和云图与 CFD 数值模拟所获结果进行了对比 分析, 如图 18 所示, 中间面高速区域均位于压力面 和外环面处, 并且在吸力面和内环处出现低速二次 流现象。LDA 测试结果的最高速度大于 CFD 仿真
值, 但 CFD 仿真预测获得的高速区范围较 LDA 所 测结果更大, 尤其是靠近内环面的高速区, LDA 测 试值与 CFD 仿真值整体上较为吻合。

\section{5 结论}

(1) 采用相对平移系数和速度误差评价不同光 束半夹角、介质折射率、平板和曲面厚度、探头位 置的折射效应影响。

（2）针对圆环壁面，分析了 $y_{0}=200$ 情况下，不 同壁厚 $d$ 、光束半夹角 $\alpha_{1}$ 、测试区域介质折射率 $n_{3}$ 和探头位置坐标 $x_{0}$ 下的 LDA 折射效应。

(3) 光束半夹角 $\alpha_{1}$ 在 $10^{\circ}$ 以内时的折射效应影 响可以忽略。

(4) 测试区域介质折射率 $n_{3}$ 和壁厚 $d$ 严重影响 测试结果, $n_{3}$ 尽可能的接近 $n_{1}, d=30 \mathrm{~mm}$, 可以获 得较小的和稳定的折射效应。

(5) 由于折射效应产生的条纹畸变, 实际测试 速度误差较理论计算有所减小, 并且由于测量体条 纹畸变的存在, 在进行流场测试前需对 LDA 测试探 头进行标定;

（6）若实际测试条件的选择达不到上述要求, 应用本文推导的曲面壁面程序, 可以对测试结果进 行相应的修正。 


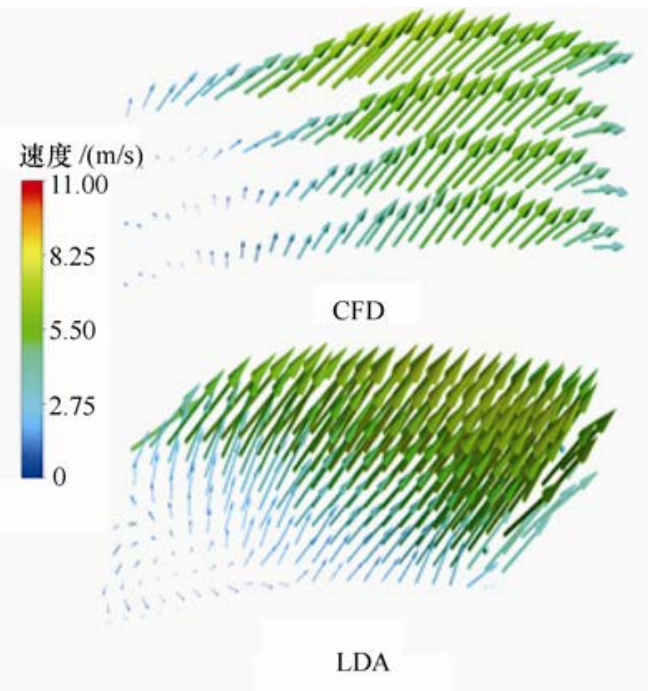

(a) 矢量图

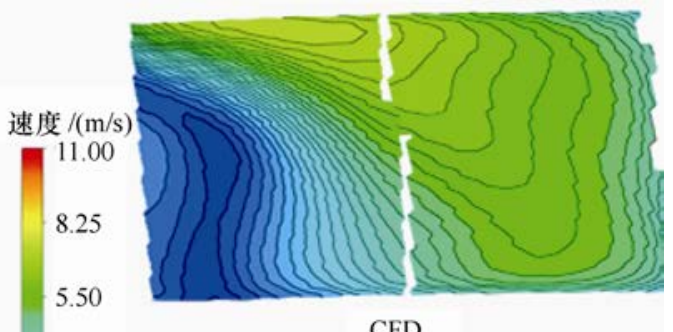

CFD

${ }_{0}^{2.75}$

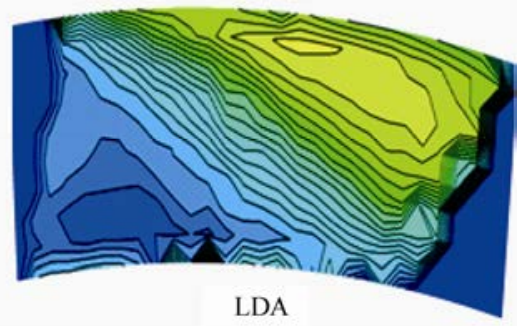

(b) 云图

图 18 中间平面速度分布图

\section{参 考 文 献}

[1] 徐兴祺. 光束穿越不同介质时 LDV 的测试及其误差分 析 [J]. 实验力学, 1992, 7(4): 329-336.

XU Xingqi. Research on the technique of LDV measurements and error analysis when the beams pass through different mediums[J]. Journal of Experimental Mechanics, 1992, 7(4): 329-336.

[2] 张莉, 陈汉平. LDA 激光探头光轴非垂直放置对测量 的影响 $[\mathrm{J}]$. 激光技术, 2001，25(4): 317-321.

ZHANG Li, CHEN Hanping. The effect of off-axis alignment of the LDA-probe on measurement[J]. Laser Technology, 2001, 25(4): 317-321.

[3] 竺晓程, 赵岩, 杜朝辉. 非正交 LDV 测量三维速度的 一些研究 $[\mathrm{J}]$. 激光技术，2002，26(6): 458-460.

ZHU Xiaocheng, ZHAO Yan, DU Zhaohui. Research on three-dimensional velocity measurement using nonorthogonalLDV[J]. Laser Technology，2002， 26(6): 458-460.
[4] 赵军, 刘宝杰, 刘火星. 机匣视窗折射对 LDV 聚焦的 影响分析[J]. 航空动力学报, 2007, 22(7): 1161-1166. ZHAO Jun, LIU Baojie, LIU Huoxing. Analysis of beam refraction effects in laser Dopplervelocimetry[J]. Journal of Aerospace Power, 2007, 22(7): 1161-1166.

[5] ZHANG Zhengji. LDA application methods : Laser doppler anemometry for fluid dynamics[M]. Berlin, Springer-Verlag Berlin Heidelberg, 2010.

[6] ZHANG Z, EISELE K. Off-axis alignment of an LDA-probe and the effect of astigmatism on measurements[J]. Experiments in Fluids, 1995,19: 89-94.

[7] ZHANG Z, EISELE K. The effect of astigmatism due to beam refractions on the formation of the measurement volume in LDA measurements[J]. Experiments in Fluids, 1996, 20: 466-471.

[8] 张超杰, 张鸣远, 卢勇, 等. 应用折射率匹配技术测 量复杂形状通道内的液体流场 $[\mathrm{J}]$. 西安交通大学学报, 2002, 36(11): 1125-1128.

ZHANG Chaojie, ZHANG Mingyuan, LU Yong, et al. Liquid velocity measurements in complex geometries using refractive index matching technique[J]. Journal of Xi’an Jiaotong University, 2002， 36(11): 1125-1128.

[9] AMINI N, HASSAN YA. An investigation of matched index of refraction technique and its application in optical measurements of fluid flow[J]. Experiments in Fluids, 2012,53: 2011-2020.

[10] STOOTS C, BECKER S, CONDIE K, et al. A large-scale matched index of refraction flow facility for LDA studies around complex geometries[J]. Experiments in Fluids, 2001, 30: 391-398.

[11] ZHANG Z. Optical guidelines and signal quality for LDA applications in circular pipes[J]. Experiments in Fluids, 2004, 37: 29-39.

[12] RISTIC S S, ILIC J T, CANTRAK D S, et al. Estimation of laser-Doppler anemometry measurement volume displacement in cylindrical pipe flow[J]. Thermal Science, 2012, 16(4): 1027-1042.

[13] DOUKELIS A, FOUNTI M, MATHIOUDAKIS K and PAPAILIOU K. Evaluation of beam refraction effects in a 3D laser Doppler anemometry system for turbomachinery applications[J]. Measurement Science Technology, 1996 (7): 922-931.

作者简介: 李春明, 男, 1964 年出生, 研究员。中国兵器工业集团首席 专家, 主要研究方向为车辆总体技术。

E-mail: chml@noveri.com

李晋(通信作者), 男, 1987 年出生, 博士, 工程师。主要研究方向为车 辆传动及流体测试技术。

E-mail: lijin929@126.com

李慎龙, 男, 1982 年出生, 研究员。主要研究方向为车辆传动技术。

E-mail: lishenlong2004@sina.com

魏巍, 男, 1978 年出生, 副教授, 硕士生研究导师。主要研究方向为车 辆传动理论和液力传动技术。

E-mail: weiweibit@bit.edu.cn 